\title{
Investigation of temperature processes in the process of interaction of technological units and production of products in a machine of bread baking
}

\author{
Nazar Sokil ${ }^{1}$, Mychailo Boiko ${ }^{1}$, Orest Serkiz ${ }^{1}$ \\ ${ }^{1}$ Department of Designing and Exploiting Machines, Lviv Polytechnic National University; \\ 12, S. Banbera Str., Lviv, 79013, Ukraine; E-mail: osnastka@ukr.net
}

\begin{abstract}
The technological process is described and the executive devices of the developed bread baking equipment are investigated. An experimental algorithm for heating the molding-baking device was experimentally investigated, established functional temperature dependencies between the matrix and heating punches. Dependence of the deformation (temperature expansion) of the interacting elements of a baking device for different temperature ranges was obtained.
\end{abstract}

Key words - grain bread, automatic machine, baking, punch, matrix, temperature, pressure.

\section{Introduction}

The profound knowledge of modern technologies and patterns of processes that are carried out during the development of food products, allows you to intensify processes, create new types of devices, develop methods for optimal process management [2].

By purpose, processes considered in food technologies, are grouped into four groups: 1 mechanical; 2 - hydromechanical; 3 - mass exchange; 4 - thermal.

It is the study of the technology of obtaining a quality product, based on the study of thermal processes in the interaction of technological units and the formation of products in the machine baking bread and devoted to the page of this work.

\section{Methodology of conducting an experiment and research results}

To obtain reliable results, a methodology for step-by-step experiments was developed:

1. Determination of the coefficients of heat transfer, linear and bulk expansion of contacting elements of the molding-baking device (two plates with punches, heated by shades and a moving matrix that contacts them). The thermoregulators that control the shades are discretely adjusting to the values of specific temperatures at an interval of 20 degrees in the range of $30 \ldots 50$ to 280 degrees. When the specified temperatures reach the pyrometer, the temperature at the control points on the plates of the punches and the matrix is fixed.

2. Since the time of reaching the temperature plate slabs of 280 degrees, the coordinate of the time is fixed and after each 5 minutes the temperature readings of the matrix control points are removed to determine the time of the device's output to the baking mode (approximately 2600). Thus, the parameters of convection of both plates are determined.

3. Repeated actions of item 1, only at control points, not temperature is measured, and linear movement (expansion) of parts of the molding-baking device.

4. When changing the temperature on the thermoregulators, when in the baking mode, at a stabilized time of baking time, they find a range of temperatures, which guarantees a highquality output.

\section{Means of control}

The following measuring instruments were used for the experiment:

- Thermostats Siemens 0 ... 24V; 500 deg .; 0.1; (included in the control panel of an automatic device for controlling the heating of eight tents with a capacity of $1.5 \mathrm{~kW}$ each); 
- Pyrometer Bosh-300 ... + 600; +/- 2 degrees;

- Digital DT9205A multimeter with thermocouple;

- Indicator lever head КИ-0,01 with a circular scale;

- Gauges 0 ... 250; 0.01;

- Temperature sensors PT-100, $D=6 \mathrm{~mm}$.

At the time of taking off the impressions, the ambient temperature in the shop was +25 degrees, humidity was $87 \%$, there were no drafts of air in the area of the machine.

Comparison of results with alternative calculations and visualized results obtained using SolidWorks software showed that they differ in the range of 5\% [1].
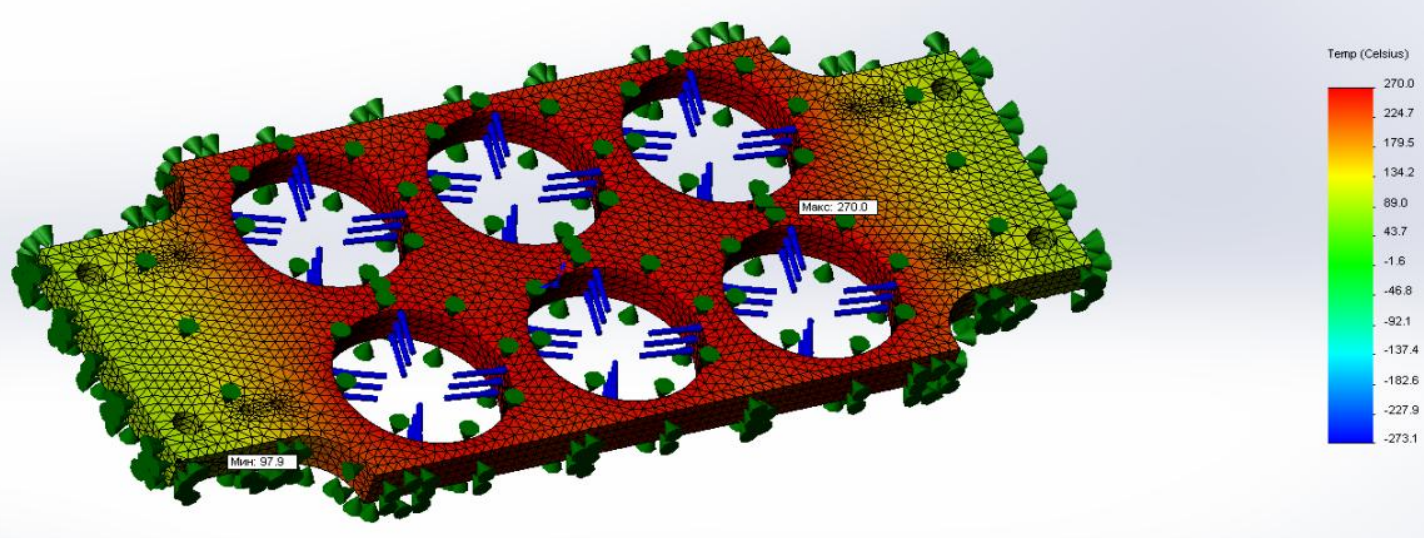

Fig. 1. Visualization of alternative results obtained using SolidWorks software.

\section{Conclusions}

1. Experimentally defined algorithm of heating the molding-baking device, to establish functional temperature dependences between the matrix and heating punches;

2. Deformation (temperature expansion) of the interacting elements of the baking device for different temperature ranges is obtained;

3. The range of temperature characteristics of the device, providing the output of a qualitative product, is determined;

4. Determine the dependencies between the force of locking the matrix with punches, the temperature of the heating and the time of baking the product;

5. Determine the optimum performance of the machine, while providing high-quality baking;

6. Compile the results of the experiment with alternative calculations and visualized results using the software SiolidWork.

\section{References}

[1] M. Dorozhovets. Working out the results of measurements: Teaching. Guide. Lviv: Publishing House of Lviv Polytechnic Nataional University, 2007.

[2] V. G. Mironchuk, I. S. Guliy, M. M. Pusancko, Eds., Equipment for processing and food industry, V. G. Mironenko Eds. Textbook. Vinnitsa: A New Book, 2007. 